\title{
Resting-state functional connectivity of the amygdala subregions in unmedicated patients with obsessive-compulsive disorder before and after cognitive behavioural therapy
}

\author{
Jian Gao, PhD; Xiangyun Yang, PhD; Xiongying Chen, PhD; Rui Liu, PhD; \\ Pengchong Wang, MA; Fanqiang Meng, MA; Zhanjiang Li, MD*; Yuan Zhou, PhD*
}

\begin{abstract}
Background: Cognitive behavioural therapy (CBT) is considered an effective first-line treatment for obsessive-compulsive disorder (OCD). However, the neural basis of CBT for OCD has not yet been elucidated. The role of the amygdala in OCD and its functional coupling with the cerebral cortex have received increasing attention, and may provide new understanding of the neural basis of CBT for OCD. Methods: We acquired baseline resting-state functional MRI (fMRI) scans from 45 unmedicated patients with OCD and 40 healthy controls; we then acquired another wave of resting-state fMRI scans from the patients with OCD after 12 weeks of CBT. We performed seed-based resting-state functional connectivity analyses of the amygdala subregions to examine changes in patients with OCD as a result of CBT. Results: Compared to healthy controls, patients with OCD showed significantly increased resting-state functional connectivity at baseline between the left basolateral amygdala and the right middle frontal gyrus, and between the superficial amygdala and the right cuneus. In patients with OCD who responded to CBT, we found decreased resting-state functional connectivity after CBT between the amygdala subregions and the visual association cortices and increased resting-state functional connectivity between the amygdala subregions and the right inferior parietal lobe. Furthermore, these changes in resting-state functional connectivity were positively associated with changes in scores on the compulsion or obsession subscales of the Yale-Brown Obsessive-Compulsive Scale. Limitations: Because of the lack of a second scan for healthy controls after 12 weeks, our results may have been confounded by other variables. Conclusion: Our findings yield insights into the pathophysiology of OCD; they also reveal the potential neural changes elicited by CBT, and thus have implications for guiding effective treatment strategies with CBT for OCD.
\end{abstract}

\section{Introduction}

Obsessive-compulsive disorder (OCD) is a chronic disabling mental disorder that affects $2 \%$ to $3 \%$ of the general population. ${ }^{1}$ It is characterized by recurrent intrusive thoughts (obsessions) and repetitive behaviours or mental acts (compulsions). Cognitive behavioural therapy (CBT) with exposure and response prevention is an effective first-line therapy for $\mathrm{OCD}$ that focuses on patients' dysfunctional thinking, emotions and behaviours. ${ }^{2}$ Although clinical symptoms can be alleviated with $\mathrm{CBT}$ in $60 \%$ to $70 \%$ of patients with OCD, those patients still experience some residual symptoms; the remaining 30\% to $40 \%$ are nonresponders. ${ }^{3}$ Thus, it is essential to elucidate the neural mechanisms that underlie OCD, as well as the potential neural basis of CBT for OCD; a better understanding of these may provide new insights that can be used to enhance existing treatments and improve treatment response in OCD.

Previous studies have indicated that the amygdala plays an important role in affective pertinence and emotional modulation. ${ }^{4,5}$ The amygdala is also a key structure in the fear circuit, which mediates the expression and extinction of conditioned fear. ${ }^{6}$ More importantly, recent studies have also pointed to the possible involvement of the amygdala in the pathogenesis of OCD, enriching our understanding of the neural mechanisms that underlie this condition. ${ }^{78}$ As well, previous studies have provided some evidence that the subregions of the amygdala may play different roles in the pathophysiology of OCD.9-11 Based on cytoarchitectonic characteristics, the amygdala can be

Correspondence to: Y. Zhou, CAS Key Laboratory of Behavioural Science, Institute of Psychology, Chinese Academy of Sciences, 16 Lincui Rd., Chaoyang District, Beijing, 100101, PR China, zhouyuan@ @sych.ac.cn; Z. Li, Beijing Anding Hospital, Capital Medical University, No. 5 Ankang Lane, Dewai Ave., Xicheng District, Beijing 100088, PR China, lizhj8@ccmu.edu.cn

${ }^{*}$ Co-corresponding authors.

Submitted May 16, 2021; Revised Aug. 3, 2021; Accepted Aug. 25, 2021

Cite as: J Psychiatry Neurosci 2021 November 16;46(6). doi: 10.1503/jpn.210084 
functionally segmented into 3 major sections: the basolateral, centromedial and superficial subregions. ${ }^{12,13}$ The basolateral amygdala is involved in fear conditioning and fear extinction learning; for example, the projection from glutamate neurons in the basolateral amygdala to glutamate and $\gamma$-aminobutyric acid neurons in the medial prefrontal cortex may have contributed to the checking symptoms of OCD in mice. ${ }^{10}$ The centromedial amygdala mediates attentional and motor responses through connectivity with the brain stem and the cortical and striatal regions; ${ }^{14}$ hyperactivity in this subregion has been reported in response to emotional face processing in patients with OCD. ${ }^{15}$ The superficial amygdala is associated with affective processes through connections with the limbic regions, ${ }^{14}$ its networks are related to the evaluation of reinforcers and risk anticipation in OCD. ${ }^{16}$ Investigating the subregions of the amygdala separately (instead of treating the amygdala as a unified structure) may provide deeper insights into how abnormalities of the amygdala contribute to the symptoms of OCD, and provide more empirical evidence for the role of the amygdala in the pathophysiology of OCD. ${ }^{17}$

In addition to the contribution of the amygdala subregions to the pathophysiology of OCD, recent studies have proposed that the functional activity of the amygdala subregions is highly associated with treatment outcomes for CBT in OCD. ${ }^{16}$ For instance, a resting-state functional MRI (fMRI) study found that the degree centrality of the right basolateral amygdala was positively correlated with response to CBT. ${ }^{16}$ Restingstate functional connectivity between the basolateral amygdala and the ventromedial prefrontal cortex also predicted treatment outcomes for CBT in OCD. ${ }^{18,19}$ However, in these studies, patients with OCD were receiving pharmacological treatment before they underwent CBT, so findings may have been confounded by the influence of medication. Moreover, these studies did not assess changes in brain activity or the functional connectivity of the amygdala after CBT, making it impossible to identify associations between changes in the circuit centred at the amygdala and treatment outcomes for CBT.

In this study, we aimed to investigate the resting-state functional connectivity of the amygdala subregions in patients with OCD, and to assess whether the intrinsic functional connectivity of the amygdala subregions was altered after CBT. We recruited a group of unmedicated patients with OCD and healthy controls. We obtained resting-state fMRI data at baseline for both groups, and after CBT in the patients with OCD. We speculated that we would find regionally dependent differences in the resting-state functional connectivity of the amygdala subregions when making the following comparisons: patients with OCD (before CBT) versus healthy controls, and patients with OCD before versus after CBT.

\section{Methods}

\section{Participants}

We recruited 50 patients with OCD from the outpatient clinics of Beijing Anding Hospital, Capital Medical University. We recruited 42 healthy controls, matched for age, sex and education level, using advertisements in the local community.
All patients with OCD were diagnosed by experienced senior psychiatrists using the Structured Clinical Interview for DSMIV Axis I Disorders (SCID-I), ${ }^{20}$ and the severity of their symptoms was assessed using the Yale-Brown Obsessive Compulsive Scale (Y-BOCS). ${ }^{21}$ Patients also completed the 17-item Hamilton Depression Rating Scale (HAM-D-17) to measure depressive symptoms. 22,23

The inclusion criteria for patients with OCD were as follows: a score of 16 or higher on the Y-BOCS scale and 18 or lower on the HAM-D-17; right-handed; aged between 18 and 45 years; no history of neurologic illnesses or other major physical diseases, Axis I psychiatric disorders other than OCD (anxiety secondary to OCD was not exclusionary) or drug or alcohol abuse; and medication-naive or medication-free for at least 4 weeks before enrolment. Of the patients with OCD, 38 had never taken medications for OCD (medication-naive) and 12 had taken medications before (selective serotonin reuptake inhibitors) but were medication-free for at least 4 weeks before enrolment. Patients were excluded if they had previous exposure to any psychotherapy.

The inclusion criteria for the healthy controls were the same as those for the patients with OCD, except that healthy controls were excluded if they had any DSM-IV diagnosis of an Axis I psychiatric disorder.

Of the participants in the present study, 24 patients with OCD and 30 healthy controls were included in a previous study ${ }^{24}$ on differences in spontaneous brain activity between patients with OCD and healthy controls. That study measured fractional amplitude of low-frequency fluctuation (fALFF) and fALFF-guided resting-state functional connectivity and was distinct from the present study.

All participants provided informed consent before participation. This study was approved by the Human Research and Ethics Committee of Beijing Anding Hospital, Capital Medical University.

\section{Treatment with $C B T$}

The patients participated in a 12-week individual CBT program, which consisted of 14 sessions based on the concepts outlined in a treatment manual for CBT in OCD (each session lasted 60 minutes). ${ }^{25,26}$ The CBT program combined cognitive interventions with exposure and response prevention. Briefly, sessions 1 and 2 involved establishment of a therapeutic alliance, collection of information and assessment; sessions 3 to 6 involved cognitive interventions such as identification of cognitive distortion, correction of the distorted cognitions and so on; sessions 7 to 12 involved preparation for and conduct of exposure and response prevention; and sessions 13 and 14 involved a review of the treatment process and relapse prevention. A more detailed description of the manual can be found in a previous report. ${ }^{27}$ All CBT therapists in the present study had rich experience in treating OCD, completed pre-study training, and received regular supervision from peers and experts to guarantee adherence to the manual. None of the patients with OCD were allowed to undergo pharmacological treatment for their obsessive-compulsive symptoms during the 12-week CBT period. Two patients with OCD who did not 
complete the 12 weeks of CBT were excluded from further analyses. We defined response to CBT as a decrease of more than $35 \%$ in total Y-BOCS score, in accordance with practice guidelines for the treatment of OCD. ${ }^{28}$ In patients with OCD, clinical symptoms were assessed by independent evaluators at baseline and 12 weeks.

\section{Brain image acquisition}

All participants were scanned using a 3.0 T magnetic resonance scanner (Magnetom Trio, Siemens) at the State Key Laboratory of Cognitive Neuroscience and Learning, Beijing Normal University, China. All participants completed MRI scans at baseline, and 48 patients with OCD also underwent scans after the 12-week CBT program. Participants were instructed to lie in a supine position with their eyes closed during scanning, but not to fall asleep or to think of anything in particular.

For resting-state functional image scanning, we used a functional sequence with the following parameters: 33 axial slices, repetition time $2000 \mathrm{~ms}$, echo time $30 \mathrm{~ms}$, flip angle $90^{\circ}$, slice thickness $3.5 \mathrm{~mm}$ with $0.6 \mathrm{~mm}$ gap, field of view $200 \times 200 \mathrm{~mm}$, in-plane resolution $64 \times 64,200$ volumes. The duration of the resting-state run was $400 \mathrm{~s}$ (6 min, $40 \mathrm{~s})$.

We also acquired high-resolution $T_{1}$-weighted images using a gradient-echo sequence with the following parameters: repetition time $2530 \mathrm{~ms}$, echo time $3.39 \mathrm{~ms}$, inversion time $1100 \mathrm{~ms}$, flip angle $15^{\circ}$, in-plane acquisition matrix $169 \times$ 196, field of view $169 \times 196 \mathrm{~mm}^{2}, 1.33 \mathrm{~mm}$ slice thickness with no interslice gap, 169 axial slices.

\section{MRI data preprocessing and analysis}

We conducted preprocessing with Data Processing and Analysis for Brain Imaging (DPABI_V3.0) software ${ }^{29}$ based on Statistical Parametric Mapping 12 (SPM 12; www.fil.ion.ucl. ac.uk/spm) and the Resting-State fMRI Data Analysis Toolkit (REST 1.8; www.restfmri.net). Preprocessing included the following steps: removal of the first 10 volumes; slice timing and spatial registration correction; $T_{1}$ map segmentation to generate grey matter, white matter and cerebrospinal fluid data; nuisance variable regression; spatial normalization (resampled to $2 \times 2 \times 2 \mathrm{~mm}^{3}$ ); and spatial smoothing (4 mm fullwidth at half-maximum Gaussian kernel). The nuisance covariates included the 5 principal components from the individual segmented cerebrospinal fluid and white matter regions, Friston's 24 motion parameters, and linear and quadratic trends. We also regressed out frame-wise motion outliers by frame-wise displacement ${ }^{30}$ using scrubbing regressors. Specifically, we identified time points with a frame-wise displacement of greater than $0.2 \mathrm{~mm}$ (plus 1 backward and 2 forward frames) as "bad" points and then modelled them as separate regressors in the regression model of the realigned resting fMRI data, removing nuisance covariates. ${ }^{31}$ Finally, we applied a band-pass filter $(0.01-0.08 \mathrm{~Hz})$ to remove lowfrequency drifts and high-frequency physiologic noise.

We excluded participants with excessive head motion. The specific criteria were as follows: more than a maximum displacement of $2.0 \mathrm{~mm}$ in either the $x, y$ or $z$ direction, or more than $2.0^{\circ}$ of angular rotation about any axis for any of the 190 volumes, based on motion parameters. Using these criteria, we excluded 3 patients with OCD and 2 healthy controls from further analysis. We also calculated the mean framewise displacement for each participant and found that none of them had a mean frame-wise displacement of more than 3 standard deviations beyond the mean of the entire sample. Therefore, we included the rest of the participants (45 patients with OCD and 40 healthy controls) in our analyses. We also used the mean frame-wise displacement of each participant to address the residual effects of motion in group-level analyses.

\section{Resting-state functional connectivity of the amygdala and its subregions}

We derived the amygdala subregions (left and right basolateral amygdala, centromedial amygdala and superficial amygdala) from the SPM Anatomy toolbox..$^{32,33}$ First, we defined the 3 subdivisions of the amygdala in each hemisphere based on stereotaxic probabilistic maps of cytoarchitectonic boundaries. Then, we then mapped them in 10 human postmortem brains, performed 3-dimensional reconstruction and mapped them to Montreal Neurological Institute space. ${ }^{12}$ We created subregion masks based on volume assignments in the maximum probability maps, which assigned each voxel exclusively to a single region. ${ }^{32}$ The subregion masks were all resampled with a voxel size of $2 \times 2 \times 2 \mathrm{~mm}^{3}$ and then taken as our seed regions for resting-state functional connectivity analyses (Figure 1). This method of defining amygdala subregions for seed-based resting-state functional connectivity

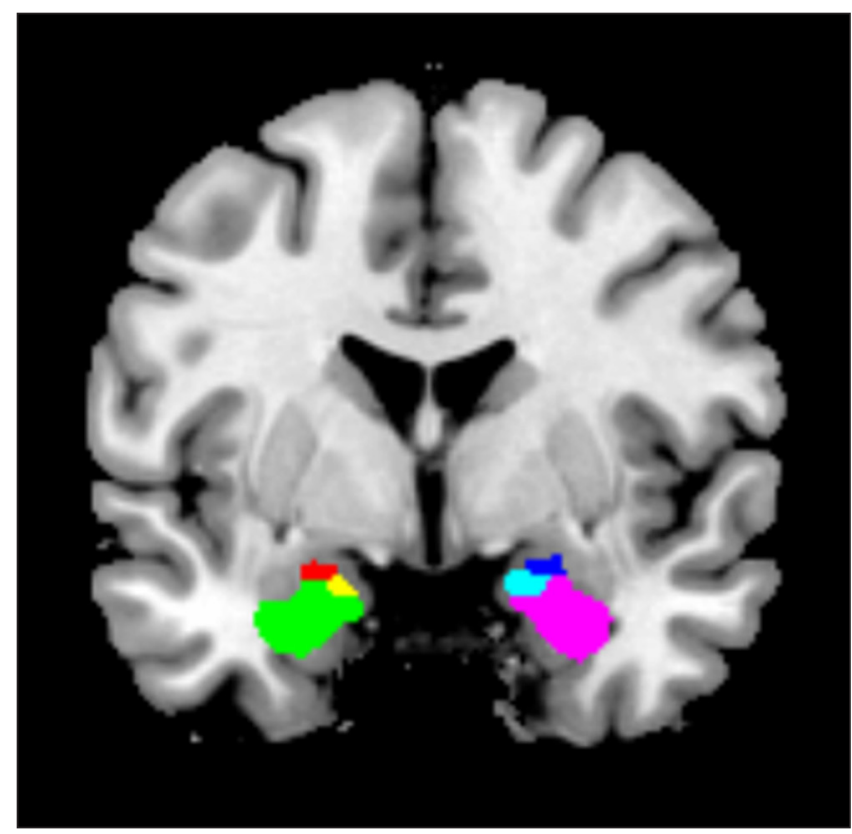

Figure 1: Subregions of the amygdala. Red: left centromedial amygdala; green: left basolateral amygdala; yellow: left superficial amygdala; dark blue: right centromedial amygdala; violet: right basolateral amygdala; light blue: right superficial amygdala. Created using the SPM Anatomy toolbox and registered to Montreal Neurological Institute space. 
analyses has been used in several previous studies. ${ }^{14,34}$ We then computed Pearson correlation coefficients between each seed and the voxels of the whole brain to create correlation maps for each seed and each participant. Finally, we transformed the correlation maps into $Z$ values using Fisher $r$-to- $Z$ transformation to improve normality.

\section{Statistical analysis}

We performed 2-sample $t$ tests and $\chi^{2}$ tests to analyze the demographic differences between patients with OCD and healthy controls. We used a paired $t$ test to compare changes in clinical symptoms between patients with OCD before and after treatment. We performed these tests in SPSS 23.0 (IBM).

For the imaging data, we conducted statistical analyses using SPM 12. First, we performed a 1-sample $t$ test for each group in a voxel-wise manner to determine the brain regions that showed significantly positive or negative correlations with each of the seed regions. Then, we performed 2-sample $t$ tests to explore differences in resting-state functional connectivity at baseline between patients with OCD and healthy controls, using age, sex and mean frame-wise displacement (head motion) as nuisance covariates. We compared differences in resting-state functional connectivity at baseline and after CBT in responders to CBT (>35\% decrease in total Y-BOCS score) using paired $t$ tests. We used 2-sample $t$ tests to determine whether resting-state functional connectivity was different in responders to CBT compared to healthy controls. The threshold of correction for multiple comparisons was $p<0.05$ (cluster-level family-wise error [FWE] corrected; individual voxel threshold $p<0.001$ ). Because the resting-state functional connectivity analysis was repeated 6 times for the amygdala subregions, we used Bonferroni correction for multiple comparisons (i.e., corrected $p<$ $0.05 / 6=0.008)$. Finally, we performed partial correlation analyses in SPSS 23.0 to explore the correlation between changes in Y-BOCS total score or subscores and averaged resting-state functional connectivity in the regions that showed significant differences before and after CBT (after Bonferroni correction) in responders to CBT (with age, sex and HAM-D-17 score as covariates; $p<0.05)$.

\section{Results}

\section{Demographic and clinical data}

Our final sample consisted of 45 patients with OCD and 40 healthy controls after excluding participants with excessive head movement and patients with OCD who did not complete the 12 weeks of CBT (Table 1). We found no differences between the 2 groups in terms of age, sex or educational level. After CBT, patients with OCD displayed improvements in OCD-related symptoms compared to baseline: Y-BOCS total scores and subscores all decreased significantly $(p<0.001)$. We identified 38 of the 45 patients with OCD as responders to CBT (total Y-BOCS score decreased $>35 \%$ ), for a response rate of $84.4 \%$, similar to response rates reported in previous studies. ${ }^{35,36}$ Additional details about the clinical characteristics of the patients with OCD can be found in Appendix 1, Table S1, available at jpn.ca.

Altered resting-state functional connectivity of the amygdala subregions at baseline in patients with $O C D$

At baseline, patients with OCD showed increased functional connectivity compared to healthy controls in the amygdalafrontal network and the amygdala-visual network. Specifically, patients with OCD had increased functional connectivity between the left basolateral amygdala and the right middle frontal gyrus (peak Montreal Neurological Institute coordinates: $x, y, z=48,8$, 38; peak $t$ : 4.37 ; cluster size: 112 voxels) and between the right superficial amygdala and the right cuneus (peak Montreal Neurological Institute coordinates: $x, y, z=2,-80,18$; peak $t$ : 4.96 ; cluster size: 122 voxels; Brodmann area 18; cluster-level $\left.p_{\mathrm{FWE}}<0.05\right)$. However, only the increased resting-state functional connectivity between the right superficial amygdala and the right cuneus survived Bonferroni correction (Figure 2).

We also compared the baseline resting-state functional connectivity of the amygdala subregions between healthy controls and responders to CBT $(n=38)$ and found similar differences in responders to CBT (Appendix 1, Table S2).

Table 1: Demographic and clinical characteristics

\begin{tabular}{|c|c|c|c|}
\hline \multirow[b]{2}{*}{ Characteristic } & \multirow{2}{*}{$\begin{array}{c}\text { Healthy } \\
\text { controls } \\
n=40\end{array}$} & \multicolumn{2}{|c|}{$\begin{array}{c}\text { OCD } \\
n=45\end{array}$} \\
\hline & & Pre-CBT & Post-CBT \\
\hline Age, yr & $28.9 \pm 6.4$ & \multicolumn{2}{|c|}{$28.7 \pm 6.7$} \\
\hline Female/male, $n$ & $18 / 22$ & \multicolumn{2}{|c|}{$20 / 25$} \\
\hline Education, yr & $13.8 \pm 2.8$ & \multicolumn{2}{|c|}{$13.7 \pm 2.9$} \\
\hline Illness duration, yr & - & \multicolumn{2}{|c|}{$7.3 \pm 6.5$} \\
\hline HAM-D score & - & $6.7 \pm 4.2$ & $5.8 \pm 5.2$ \\
\hline Y-BOCS total score & - & $23.6 \pm 5.9$ & $10.3 \pm 5.6$ \\
\hline Y-BOCS obsession subscale score & - & $12.2 \pm 4.8$ & $5.1 \pm 3.2$ \\
\hline Y-BOCS compulsion subscale score & - & $11.4 \pm 3.8$ & $5.2 \pm 3.4$ \\
\hline \multicolumn{4}{|c|}{$\begin{array}{l}\mathrm{CBT}=\text { cognitive behavioural therapy; HAM-D = Hamilton Depression Rating Scale; OCD = obsessive- } \\
\text { compulsive disorder; Y-BOCS = Yale-Brown Obsessive Compulsive Scale. } \\
\text { Values are mean + standard deviation, unless otherwise indicated. }\end{array}$} \\
\hline
\end{tabular}


Altered resting-state functional connectivity of the amygdala subregions in responders to CBT

The main aim of the present study was to investigate changes in resting-state functional connectivity of the amygdala subregions in responders to CBT compared to baseline $(n=38)$. After treatment, responders to CBT showed decreased restingstate functional connectivity between the left centromedial amygdala and the left middle temporal gyrus and between the right centromedial amygdala and the left middle occipital gyrus. We also found decreased resting-state functional connectivity after treatment in responders to CBT between the left basolateral amygdala and the left middle temporal gyrus, between the left inferior temporal gyrus and the left middle occipital gyrus, between the right basolateral amygdala and the left middle occipital gyrus, and between the right superficial amygdala and the right middle temporal gyrus. Conversely, we found that responders to CBT showed increased resting-state functional connectivity between the right inferior parietal lobule and 3 subregions of the amygdala (the left centromedial amygdala and the left and right superficial amygdala) after CBT (cluster-level $p_{\mathrm{FWE}}<0.05$; Figure 3, Table 2). Some of these alterations survived Bonferroni correction (Table 2; cluster-level $p_{\mathrm{FWE}}<0.008$ ).
To explore whether resting-state functional connectivity in responders to CBT was different from healthy controls, we compared resting-state functional connectivity for each amygdala subregion between responders to CBT $(n=38)$ after 12 weeks of treatment and healthy controls at baseline. We found no significant differences between the 2 groups (cluster-level $p_{\mathrm{FWE}}>0.05$; Appendix 1, Figure S1).

\section{Association between changes in resting-state functional connectivity of the amygdala subregions and the efficacy of $C B T$ in responders to $C B T$}

We examined the association between changes in the resting-state functional connectivity of the amygdala subregions and changes in clinical symptoms after CBT using partial correlation analyses in responders to CBT $(n=38)$. We found that decreased resting-state functional connectivity between the right centromedial amygdala and the left middle occipital gyrus was positively correlated with a reduction in Y-BOCS compulsion score. Decreased restingstate functional connectivity between the left basolateral amygdala and the left inferior temporal gyrus was positively correlated with a reduction in Y-BOCS obsession score (Figure 4).
A
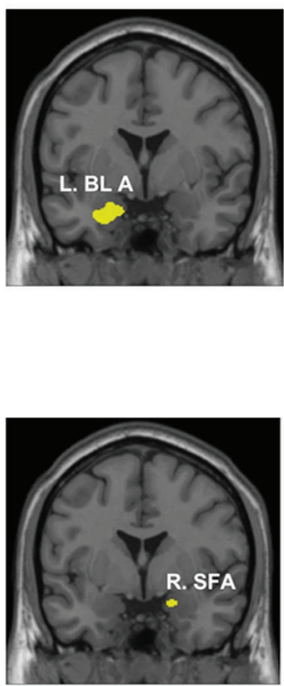

Seed
B
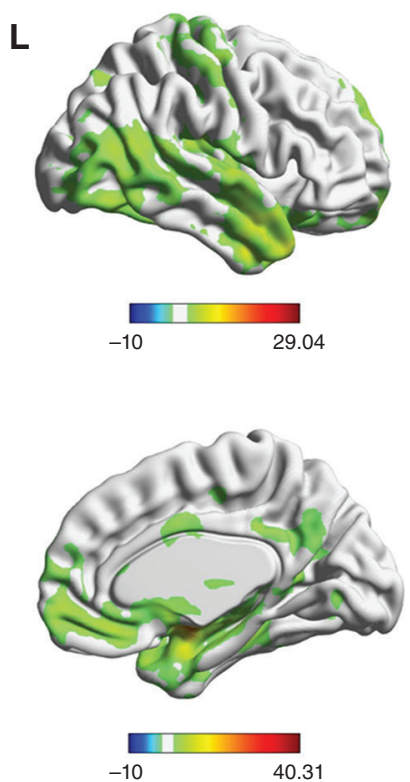

Healthy controls
C
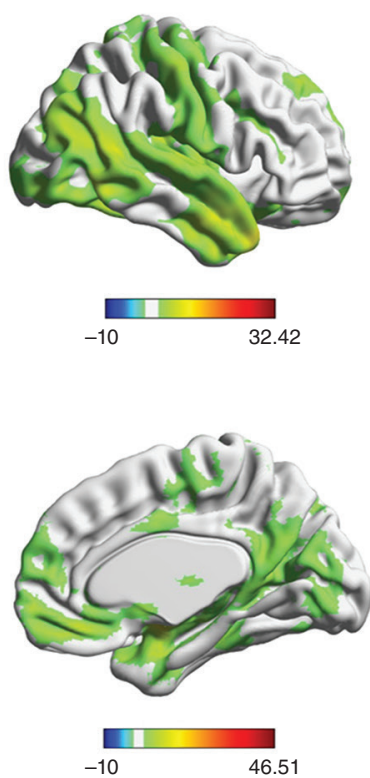

OCD
D
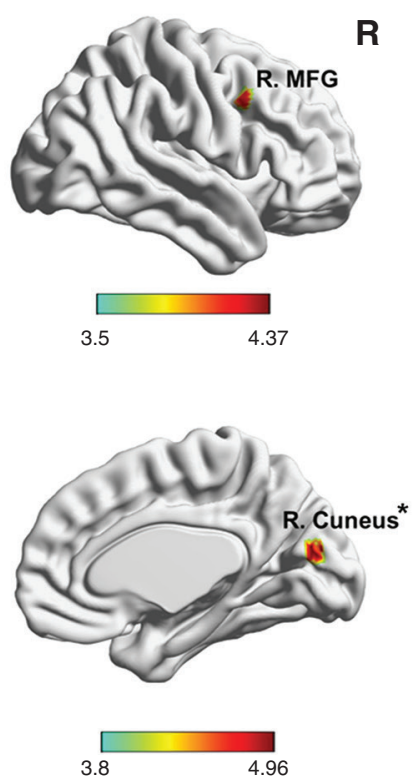

Healthy controls v. OCD

Figure 2: Resting-state functional connectivity of the amygdala subregions that showed significant differences at baseline in patients with OCD compared to healthy controls. (A) Seed regions: left basolateral amygdala and right superficial amygdala. (B and C) Resting-state functional connectivity in (B) healthy controls and (C) patients with OCD. (D) Regions in which we found significant differences in resting-state functional connectivity with the seed region (cluster-level $p_{\mathrm{FWE}}<0.05$ ). ${ }^{*}$ Survived Bonferroni correction. BLA = basolateral amygdala; FWE = family-wise error; MFG = middle frontal gyrus; OCD = obsessive-compulsive disorder; SFA = superficial amygdala. 


\section{Discussion}

To our knowledge, this study was the first to explore alterations in the circuit centred at the amygdala subregions before and after CBT for OCD. We showed an association between resting-state functional connectivity in these subregions and improvement in clinical symptoms in patients with OCD. At baseline, we found increased functional connectivity between the amygdala subregions and the frontal networks (the right middle frontal gyrus), and between the amygdala subregions and the visual cortex (the right cuneus) in patients with OCD compared to healthy controls. This finding indicates that the amygdala may contribute to the pathophysiology of OCD via the perceptual processing of emotional stimuli, a finding that would enrich the pathology model of OCD. More importantly, after 12 weeks of CBT, resting-state functional connectivity between the amygdala subregions and the regions in the visual association cortices decreased in responders to CBT. These changes were significantly associated with improvements in obsessive-compulsive symptoms.

Altered resting-state functional connectivity of the amygdala subregions at baseline in patients with OCD

We found increased functional connectivity between the left basolateral amygdala and the right middle frontal gyrus in

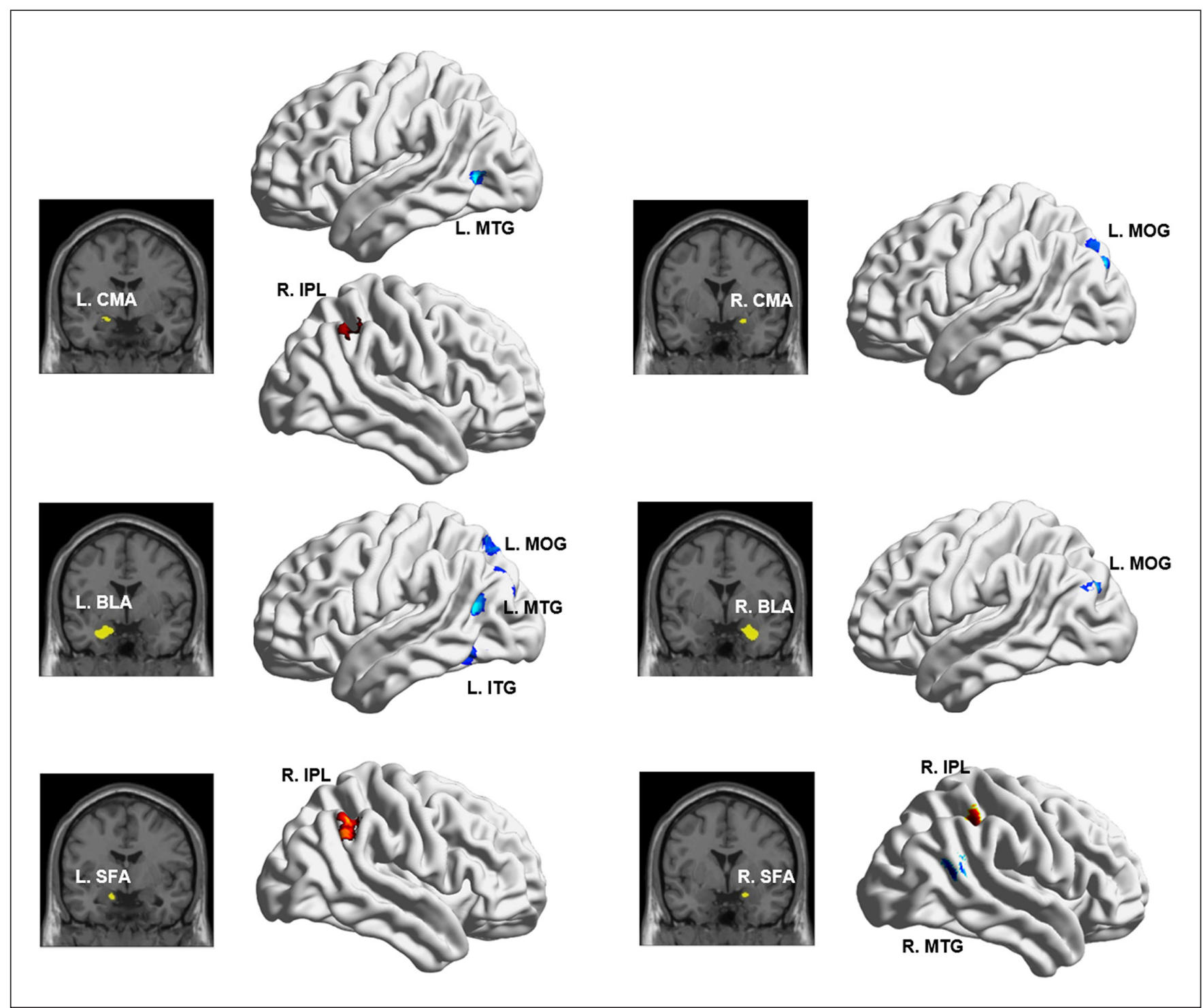

Figure 3: Altered resting-state functional connectivity of the amygdala subregions in patients with OCD who responded to CBT, before and after treatment (cluster-level $p_{\text {FWE }}<0.05$ ). The red regions are locations where functional connectivity with the amygdala subregions was increased in responders to CBT after treatment (compared to before treatment). The blue regions are locations where functional connectivity with the amygdala subregions was decreased in responders to CBT after treatment. BLA = basolateral amygdala; CBT = cognitive behavioural therapy; CMA = centromedial amygdala; FWE = family-wise error; IPL = inferior parietal lobule; ITG = inferior temporal gyrus; $\mathrm{MOG}=$ middle occipital gyrus; MTG = middle temporal gyrus; OCD = obsessive-compulsive disorder; SFA = superficial amygdala. 
unmedicated patients with OCD, indicating an abnormality in the amygdala-frontal network. Previous functional studies have found that connectivity of the amygdala and the prefrontal cortex subserved emotion regulation, and the influence of the amygdala on the right middle frontal gyrus was positively correlated with neuroticism. ${ }^{37-39}$ Compared to healthy controls, patients with OCD have shown higher scores in neuroticism, ${ }^{40,41}$ which could increase their susceptibility to negative emotion and lead to difficulties in emotion regulation. ${ }^{42,43}$ One fMRI study also indicated that connectivity between the basolateral amygdala and the right middle frontal gyrus might underlie emotional appraisal or expression and regulation. ${ }^{44}$ Similarly, our finding that patients with
OCD showed increased resting-state functional connectivity between the basolateral amygdala and the right middle frontal gyrus at baseline compared to healthy controls suggests that patients with OCD may experience dysfunctional emotional appraisal and difficulty regulating negative emotion.

We also observed alterations in resting-state functional connectivity between the right superficial amygdala and the right cuneus in patients with OCD. The cuneus, as part of the visual cortex, is involved in visual spatial processing and receives visual information from the contralateral retina, which is then projected to extrastriate cortices (Brodmann areas 18 and 19). ${ }^{45}$ Previous studies have shown that the functional coupling between the amygdala (involved in regulating

\begin{tabular}{|c|c|c|c|c|c|c|}
\hline Amygdala seed & Brain region & Voxels & $\begin{array}{c}\text { Brodmann } \\
\text { area }\end{array}$ & $\begin{array}{c}\text { MNI coordinates, } \\
x, y, z\end{array}$ & $t$ & $p_{\mathrm{FWE}}$ \\
\hline \multicolumn{7}{|l|}{ Pre-CBT > post-CBT } \\
\hline Left centromedial amygdala & Left middle temporal gyrus & 107 & 37 & $-50,-68,2$ & 5.13 & 0.009 \\
\hline Right centromedial amygdala & Left middle occipital gyrus & 120 & 19 & $-30,-82,28$ & 4.64 & $0.005^{\star}$ \\
\hline \multirow[t]{3}{*}{ Left basolateral amygdala } & Left inferior temporal gyrus & 158 & 19 & $-50,-64,-6$ & 4.46 & $0.001^{*}$ \\
\hline & Left middle temporal gyrus & 105 & $39 / 19$ & $-38,-62,14$ & 5.45 & 0.011 \\
\hline & Left middle occipital gyrus & 427 & $19 / 39$ & $-30,-78,30$ & 5.09 & $<0.001^{*}$ \\
\hline Right basolateral amygdala & Left middle occipital gyrus & 85 & 39 & $-34,-80,28$ & 4.59 & 0.034 \\
\hline Right superficial amygdala & Right middle temporal gyrus & 88 & 39 & $-50,-60,18$ & 5.15 & 0.027 \\
\hline \multicolumn{7}{|l|}{ Pre-CBT < post-CBT } \\
\hline Left centromedial amygdala & Right inferior parietal lobule & 291 & 40 & $42,-44,36$ & 4.83 & $<0.001^{*}$ \\
\hline Left superficial amygdala & Right inferior parietal lobule & 219 & 40 & $58,-48,48$ & 5.58 & $<0.001^{*}$ \\
\hline Right superficial amygdala & Right inferior parietal lobule & 125 & 40 & $40,-46,44$ & 5.03 & $0.002^{*}$ \\
\hline
\end{tabular}
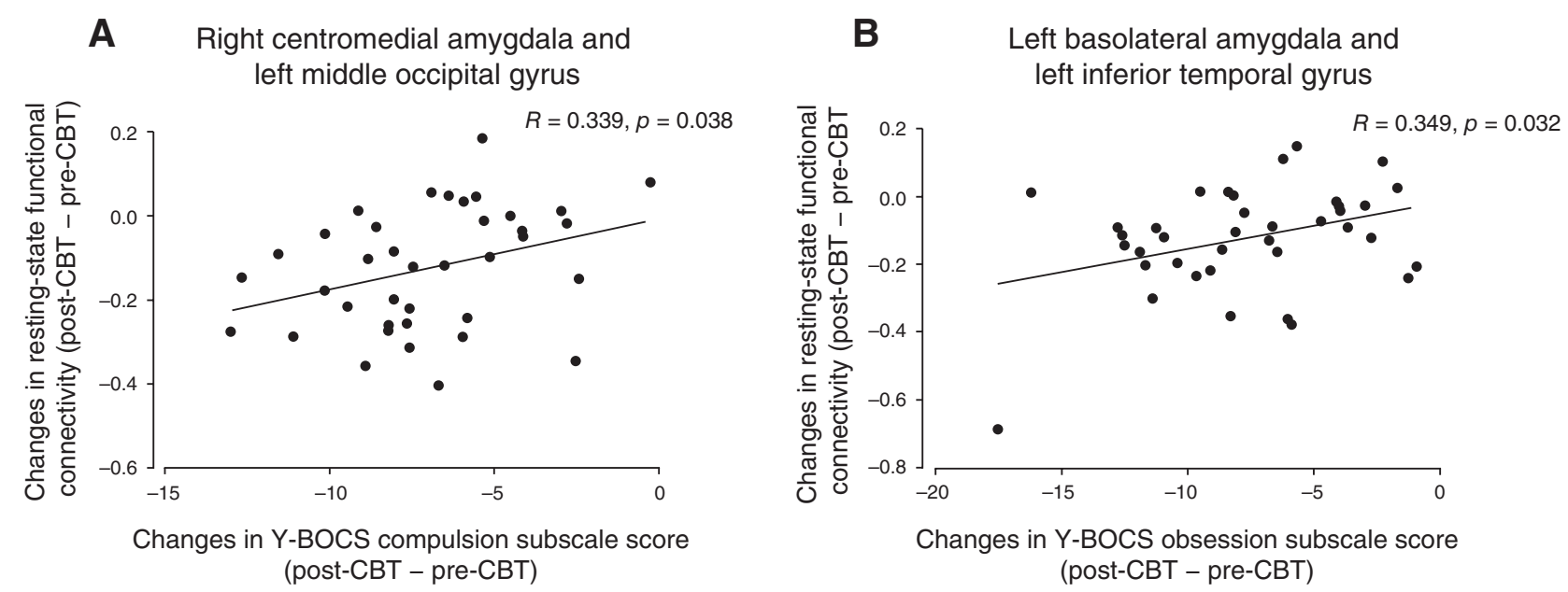

Figure 4: Correlations between changes in functional connectivity in amygdala subregions and symptom improvement in responders to CBT. (A) Positive correlation between changes in resting-state functional connectivity after CBT (right centromedial amygdala and left middle occipital gyrus) and a reduction in Y-BOCS compulsion score in responders to CBT. (B) Positive correlation between changes in resting-state functional connectivity after CBT (left basolateral amygdala and inferior temporal gyrus) and a reduction in Y-BOCS obsession score in responders to CBT. $\mathrm{CBT}=$ cognitive behavioural therapy; Y-BOCS, Yale-Brown Obsessive Compulsive Scale. 
"classical" emotions) and the extrastriate visual regions was altered when patients with OCD viewed stimuli with a negative valence. ${ }^{5,8}$ Another study noted that functional connectivity between the superficial amygdala and the visual areas was increased during fear stimuli (compared with joy), suggesting that the functional coupling between the superficial amygdala and the visual areas may be vital to visual alertness. ${ }^{46}$ Taken together, our results complement previous studies by showing that dysfunctional coupling between the superficial amygdala and the visual cortex plays an important role in visual processing, especially in processing negative, fear-provoking stimuli for patients with OCD.

\section{Altered resting-state functional connectivity of the amygdala subregions in responders to $C B T$}

We further explored changes in resting-state functional connectivity of the amygdala subregions in patients with OCD who responded to CBT, and we observed decreased restingstate functional connectivity between the amygdala subregions and the visual association cortices, including the temporal cortex and the occipital cortex. In particular, CBT decreased the functional connectivity between the right centromedial amygdala and the middle occipital gyrus. Previous neuroimaging studies have indicated that the amygdala was highly activated with the visual association cortices, which mainly included the middle occipital gyrus in the processing of emotional information. ${ }^{47}$ Another resting-state fMRI study ${ }^{48}$ found that the hypothalamic neuropeptide oxytocin could decrease the restingstate functional connectivity between the right centromedial amygdala and the right middle occipital gyrus. The authors suggested that the modulatory effects of oxytocin on the networks related to emotional face processing are mediated by the functional connectivity of the centromedial amygdala. As well, based on the evidence for the different functions of the subregions of amygdala in emotion processing, ${ }^{49}$ the centromedial amygdala was preferentially sensitive to highly arousing negative emotional stimuli. ${ }^{50}$ Thus, our results may suggest that CBT could regulate the emotional processing of negative content in patients with OCD by reducing the functional coupling between the centromedial amygdala and the middle occipital gyrus. We also found that decreased resting-state functional connectivity was associated with a reduction in the compulsive behaviour scores in patients with OCD who responded to $\mathrm{CBT}$. This modulatory influence of $\mathrm{CBT}$ on the connectivity of the centromedial amygdala may provide a neural basis for CBT in decreasing compulsive repetitive behaviours in patients with OCD.

We found additional changes in the connectivity of the amygdala subregions after CBT, as evidenced by decreased connections from the basolateral amygdala to the visual association cortices (the inferior and middle temporal gyrus and middle occipital gyrus). Previous neuroimaging studies have indicated that the activation of the amygdala may influence the early recruitment of the ventral visual stream, including the occipital gyrus. The occipital gyrus becomes sensitive to disorder-relevant stimuli and relays detection to the middle temporal cortex, which in turn upregulates activity in the amygdala. ${ }^{51,52} \mathrm{~A}$ recent meta-analysis found that activation of regions including the bilateral amygdala, the middle temporal cortex and the middle occipital cortex was significantly increased during emotion processing in OCD. ${ }^{53}$ Based on these findings, we can assume that the amygdala has close functional coupling with the visual association cortices, including the temporal and occipital cortices, and that functional connectivity among these regions is likely to reflect their involvement in the emotional processing of disorderrelevant stimuli in OCD. ${ }^{54,55}$ Moreover, dysfunction of the coupling between the basolateral amygdala and the visual association areas may cause a perceptual bias effect in those whose perception of ongoing tasks is easily influenced by task-irrelevant stimuli, and this may lead to impaired integration of the perceptual and emotional processes. ${ }^{56,57}$ In our study, we found that CBT could dampen the increased resting-state functional connectivity between the visual association cortices and the basolateral amygdala in OCD, and that such altered resting-state functional connectivity was positively associated with improvements in obsessivecompulsive symptoms in patients with OCD who responded to CBT. This finding was also in line with previous results in which CBT reduced hyperactivity in brain regions associated with emotion processing, such as the amygdala and the middle temporal gyrus. More importantly, these neural changes adjusted by CBT could also help patients with anxiety disorders reduce their clinical symptoms. ${ }^{58,59}$ Taken together, our findings suggest that CBT modulates emotional processing in patients with OCD by integrating visual and emotional signals related to higher cognitive processes such as reappraisal, $^{60}$ and by decreasing the perceptual bias toward threatening stimuli that may easily induce symptoms.

We also found increased resting-state functional connectivity between the subregions of the amygdala (centromedial amygdala and superficial amygdala) and the right inferior parietal lobule in patients with OCD who were responders to CBT. A previous study indicated that the inferior parietal lobule may integrate emotional information from the limbic regions (e.g., amygdala) and constitute "hubs" or "switches" in the processing of emotional conflict. ${ }^{61}$ Resting-state functional connectivity between the superficial amygdala and the inferior parietal lobule has also been associated with emotional lability scores in patients with attention-deficit/hyperactivity disorder, which suggests emotional self-regulation dysfunction. ${ }^{62}$ However, a recent study reported that patients with OCD showed decreased resting-state functional connectivity between the left amygdala and the left inferior parietal cortex after 4 weeks of cognitive-coping therapy. ${ }^{36}$ The inconsistency between that study and the present study can be attributed to patient characteristics, the content of psychotherapy and the duration of the observation window. In the present study, patients were medication-naïve or medication-free for at least 4 weeks before enrolment, and patients were excluded if they had previous exposure to any psychotherapy. In the study by Zhao and colleagues, ${ }^{36}$ most of the participants $(21 / 26)$ were taking selective serotonin reuptake inhibitors when they were enrolled in the study. Such a difference in medication status may have influenced resting-state functional connectivity, as 
suggested in a previous study. ${ }^{63}$ The psychotherapy used in the present study was also different from that used in the study by Zhao and colleagues. ${ }^{36}$ In our study, CBT involved 5 sessions of behavioural intervention (i.e., exposure and response prevention) in addition to the cognitive interventions. The study by Zhao and colleagues, ${ }^{36}$ used cognitive-coping therapy (a novel emotion- and motivation-focused therapy for OCD). This difference between the 2 studies suggests that different psychotherapy techniques may induce different functional brain changes, an interesting and important question to be addressed in future studies. Finally, the treatment period used in the 2 studies was different. Psychotherapy lasted 12 weeks in the present study, but only 4 weeks in the study by Zhao and colleagues, ${ }^{36}$ leading to differences in the observation window. It is possible that the resting-state functional connectivity of the amygdala could change dynamically across the treatment period. Future studies can test this possibility by scanning at multiple time points during treatment (e.g., pre-treatment, mid-treatment and post-treatment). Still, in spite of the differences between the present study and that of Zhao and colleagues, ${ }^{36}$ both suggested that a functional coupling between the amygdala and the parietal lobe - associated with the processing of emotional information and emotional self-regulation - might be in involved in OCD and could be altered by psychotherapy.

\section{Implications of the present study}

This study provided detailed evidence for the functional connectivity of amygdala subregions that could contribute to the pathological mechanisms of OCD and might be significantly altered by CBT. However, differences in resting-state functional connectivity of the amygdala subregions between patients with OCD and healthy controls at baseline did not overlap with changes in resting-state functional connectivity in responders to CBT. These results suggest that the neural basis of treatment response might not be the neural basis of the etiology of OCD, or the manifestation of a neuropsychiatric disorder that tends to highlight the differences between patients and healthy controls. This concept is in line with the findings of previous studies that have explored the mechanisms of CBT for social anxiety disorder. ${ }^{64-66}$ We also found that the resting-state functional connectivity of the amygdala subregions could change after CBT and further revealed its relationship with treatment efficacy (i.e., changes in symptom severity). We speculate that resting-state functional connectivity between the amygdala subregions and the visual association cortices in patients with OCD may be associated with a perceptual bias effect and lead to dysfunctional emotion regulation, which in turn translates to the therapeutic effects of CBT. This finding echoes the view of Reggente and colleagues, ${ }^{67}$ who raised the possibility that one of the effects of CBT might be to significantly adjust the interplay between the visual network and the amygdala. To the best of our knowledge, this adjustment has not yet been proven with empirical evidence. However, our findings support this view and suggest that the functional coupling of the amygdala subregions with the visual association cortices could serve as the underlying neural basis for the effect of CBT for OCD. We also found no significant group differences in baseline resting-state functional connectivity between responders to CBT and healthy controls. This finding suggests that the resting-state functional connectivity of the amygdala in patients with OCD could be normalized with treatment. However, this finding should be interpreted with caution because of our small sample size and the lack of a second scan for the healthy controls.

\section{Limitations}

Our findings are promising, but the present study had several limitations. The control group in our study might have been inadequate because of the lack of a 12-week waitlist condition. Our study recruited a healthy control group instead of a waitlist group, because putting patients with OCD in a placebo group for nearly 3 months would have been unlikely to receive approval from our local ethics committee. In addition, we did not conduct a second resting-state fMRI scan after 12 weeks in the healthy control group, so we were unable to draw conclusions about whether the changes in patients with OCD were specific to CBT or reflected a better general prognostic outcome. In other words, the results may have been confounded by other variables such as scanner noise from the time interval. Still, the present study provides detailed information about changes in the neural connectivity of the amygdala subregions after CBT in patients with OCD, and in this way it may still help guide future study to explore the therapeutic mechanisms of CBT by focusing on the function of the amygdala and its subregions. Although we tried to reduce heterogeneity among the patients with OCD by recruiting people who were unmedicated, our findings may still have been confounded by heterogeneity in OCD subtypes, which may have different neural bases and different neural mechanisms for the effects of CBT. ${ }^{68}$ Future studies should focus on OCD subtypes. Finally, we lacked objective measures of emotional processing in our patient group, so we were unable to draw definitive conclusions about the relationship between altered functional connectivity and changes in emotion processing after CBT. Further research could incorporate neuropsychological testing or task-based fMRI to confirm the hypotheses formulated here.

\section{Conclusion}

Our study showed altered resting-state functional connectivity centred on the amygdala subregions in OCD and provides relatively detailed evidence for the underlying mechanisms of CBT for OCD. The decreased functional connectivity we found between the amygdala subregions and the visual association cortices may reflect an effect of CBT on emotional processing. These findings enrich the classic corticostriatal models of OCD pathophysiology by adding more empirical evidence for the amygdala, and they suggest a potential neural basis for CBT, which might be useful for guiding the development of effective treatment strategies for OCD in the future. 
Acknowledgements: This work was supported by the National Natural Science Foundation of China (81271493), the Beijing Natural Science Foundation (7122082) and the Beijing Municipal Administration of Hospitals Clinical Medicine Development of Special Funding Support (XMLX202129). We are grateful to Mrs. Yuening Jin for her help in English editing.

Affiliations: From the National Clinical Research Center for Mental Disorders \& Beijing Key Laboratory of Mental Disorders, Beijing Anding Hospital, Capital Medical University, Beijing, China (Gao, Yang, Chen, Liu, Wang, Meng, Li, Zhou); the Advanced Innovation Center for Human Brain Protection, Capital Medical University, Beijing, China (Gao, Yang, Chen, Liu, Wang, Meng, Li); the CAS Key Laboratory of Behavioral Science, Institute of Psychology, Beijing, China (Zhou); the Department of Psychology, University of Chinese Academy of Sciences, Beijing, China (Zhou); and the Magnetic Resonance Imaging Research Center, Institute of Psychology, Chinese Academy of Sciences, Beijing, China (Zhou)

\section{Competing interests: None declared.}

Contributors: Z. Li and Y. Zhou designed the study. X. Yang, P. Wang and F. Meng acquired the data, which J. Gao, X. Chen, R. Liu and Y. Zhou analyzed. J. Gao wrote the article, which X. Yang, X. Chen, R. Liu, P. Wang, F. Meng, Z. Li and Y. Zhou reviewed. All authors approved the final version to be published and can certify that no other individuals not listed as authors have made substantial contributions to the paper.

Ethical standards: The authors note that all procedures contributing to this work comply with the ethical standards of the relevant national and institutional committees on human experimentation and with the Helsinki Declaration of 1975, as revised in 2008.

Content licence: This is an Open Access article distributed in accordance with the terms of the Creative Commons Attribution (CC BY-NC-ND 4.0) licence, which permits use, distribution and reproduction in any medium, provided that the original publication is properly cited, the use is noncommercial (i.e., research or educational use), and no modifications or adaptations are made. See: https:/ / creativecommons.org/licenses/by-nc-nd/4.0/

\section{References}

1. Ruscio AM, Stein DJ, Chiu WT, et al. The epidemiology of obsessivecompulsive disorder in the National Comorbidity Survey Replication. Mol Psychiatry 2010;15:53-63.

2. Ponniah K, Magiati I, Hollon SD. An update on the efficacy of psychological therapies in the treatment of obsessive-compulsive disorder in adults. J Obsessive Compuls Relat Disord 2013;2:207-18.

3. Olatunji BO, Davis ML, Powers MB, et al. Cognitive-behavioral therapy for obsessive-compulsive disorder: a meta-analysis of treatment outcome and moderators. J Psychiatr Res 2013;47:33-41.

4. Sander D, Grafman J, Zalla T. The human amygdala: an evolved system for relevance detection. Rev Neurosci 2003;14:303-16.

5. Wendt J, Weike AI, Lotze M, et al. The functional connectivity between amygdala and extrastriate visual cortex activity during emotional picture processing depends on stimulus novelty. Biol Psychol 2011;86:203-9.

6. Phelps EA, Delgado MR, Nearing KI, et al. Extinction learning in humans: role of the amygdala and vmPFC. Neuron 2004;43:897-905.

7. Milad MR, Rauch SL. Obsessive-compulsive disorder: beyond segregated cortico-striatal pathways. Trends Cogn Sci 2012;16:43-51.

8. Rus OG, Reess TJ, Wagner G, et al. Functional and structural connectivity of the amygdala in obsessive-compulsive disorder. Neuroimage Clin 2016;13:246-55.

9. Subirà M, Cano M, de Wit SJ, et al. Structural covariance of neostriatal and limbic regions in patients with obsessive-compulsive disorder. J Psychiatry Neurosci 2016;41:115-23.

10. Sun T, Song Z, Tian Y, et al. Basolateral amygdala input to the medial prefrontal cortex controls obsessive-compulsive disorderlike checking behavior. Proc Natl Acad Sci U S A 2019;116:3799-804.
11. Via E, Cardoner N, Pujol J, et al. Amygdala activation and symptom dimensions in obsessive-compulsive disorder. Br J Psychiatry 2014;204:61-8.

12. Amunts $\mathrm{K}$, Kedo O, Kindler M, et al. Cytoarchitectonic mapping of the human amygdala, hippocampal region and entorhinal cortex: intersubject variability and probability maps. Anat Embryol (Berl) 2005;210:343-52

13. Bzdok D, Laird AR, Zilles K, et al. An investigation of the structural, connectional, and functional subspecialization in the human amygdala. Hum Brain Mapp 2013;34:3247-66.

14. Zheng D, Chen J, Wang X, et al. Genetic contribution to the phenotypic correlation between trait impulsivity and resting-state functional connectivity of the amygdala and its subregions. Neuroimage 2019;201:115997.

15. Via E, Cardoner N, Pujol J, et al. Amygdala activation and symptom dimensions in obsessive-compulsive disorder. Br J Psychiatry 2014; 204:61-8.

16. Gottlich M, Kramer UM, Kordon A, et al. Resting-state connectivity of the amygdala predicts response to cognitive behavioral therapy in obsessive compulsive disorder. Biol Psychol 2015;111:100-9.

17. Picó-Pérez M, Ipser J, Taylor P, et al. Intrinsic functional and structural connectivity of emotion regulation networks in obsessivecompulsive disorder. Depress Anxiety 2019;36:110-20.

18. Fullana MA, Zhu X, Alonso $P$, et al. Basolateral amygdala-ventromedial prefrontal cortex connectivity predicts cognitive behavioural therapy outcome in adults with obsessive-compulsive disorder. J Psychiatry Neurosci 2017;42:378-85.

19. Norman LJ, Mannella KA, Yang H, et al. Treatment-specific associations between brain activation and symptom reduction in OCD following CBT: a randomized fMRI trial. Am I Psychiatry 2021;178:39-47.

20. First MB, Spitzer RL, Gibbon M, et al. Structured clinical interview for DSM-IV-TR Axis I disorders (SCID-I) research. New York: New York State Psychiatric Institute, Biometrics Department; 2002.

21. Goodman WK, Price LH, Rasmussen SA, et al. The Yale-Brown Obsessive Compulsive Scale. I. Development, use, and reliability. Arch Gen Psychiatry 1989;46:1006-11.

22. Ballesteros J, Bobes J, Bulbena A, et al. Sensitivity to change, discriminative performance, and cutoff criteria to define remission for embedded short scales of the Hamilton depression rating scale (HAMD). I Affect Disord 2007;102:93-9.

23. Hamilton M. Development of a rating scale for primary depressive illness. Br J Soc Clin Psychol 1967;6:278-96.

24. Gao J, Zhou Y, Yang X, et al. Abnormalities within and beyond the cortico-striato-thalamo-cortical circuitry in medication-free patients with OCD revealed by the fractional amplitude of low-frequency fluctuations and resting-state functional connectivity. Neurosci Lett 2019;712:134449.

25. Whittal ML, Thordarson DS, McLean PD. Treatment of obsessivecompulsive disorder: cognitive behavior therapy vs. exposure and response prevention. Behav Res Ther 2005;43:1559-76.

26. Wilson KA, Chambless DL. Cognitive therapy for obsessivecompulsive disorder. Behav Res Ther 2005;43:1645-54.

27. Yang XY, Sun J, Luo J, et al. Regional homogeneity of spontaneous brain activity in adult patients with obsessive-compulsive disorder before and after cognitive behavioural therapy. J Affect Disord 2015;188:243-51.

28. Koran LM, Hanna GL, Hollander E, et al. Practice guideline for the treatment of patients with obsessive-compulsive disorder. Am J Psychiatry 2007;164:5-53.

29. Yan CG, Wang XD, Zuo XN, et al. DPABI: Data processing and analysis for (resting-state) brain imaging. Neuroinformatics 2016;14: 339-51.

30. Power JD, Barnes KA, Snyder AZ, et al. Spurious but systematic correlations in functional connectivity MRI networks arise from subject motion. Neuroimage 2012;59:2142-54.

31. Yan CG, Cheung B, Kelly C, et al. A comprehensive assessment of regional variation in the impact of head micromovements on functional connectomics. Neuroimage 2013;76:183-201.

32. Eickhoff SB, Stephan KE, Mohlberg H, et al. A new SPM toolbox for combining probabilistic cytoarchitectonic maps and functional imaging data. Neuroimage 2005;25:1325-35.

33. Eickhoff SB, Heim S, Zilles K, et al. Testing anatomically specified hypotheses in functional imaging using cytoarchitectonic maps. Neuroimage 2006;32:570-82. 
34. Kerestes R, Chase HW, Phillips ML, et al. Multimodal evaluation of the amygdala's functional connectivity. Neuroimage 2017;148:219-29.

35. Torun G, Bjarne $\mathrm{H}$, Svein $\mathrm{H}$, et al. Three-week inpatient treatment of obsessive-compulsive disorder: a 6-month follow-up study. Front Psychol 2018;9:620.

36. Zhao Z, Wang CH, Ma JD, et al. Decreased left amygdala functional connectivity by cognitive-coping therapy in obsessive-compulsive disorder. Mol Psychiatry 2021 May 7 [Epub ahead of print]. doi: 10.1038/s41380-021-01131-z.

37. Cremers HR, Aleman A, Demenscu L, et al. Neuroticism modulates amygdala-medial prefrontal connectivity in response to negative emotional faces. Neuroimage 2010;49:963-70.

38. Pang Y, Cui Q, Wang Y, et al. Extraversion and neuroticism related to the resting-state effective connectivity of amygdala. Sci Rep 2016;6:35484.

39. Tzschoppe J, Nees F, Banaschewski T, et al. Aversive learning in adolescents: modulation by amygdala-prefrontal and amygdalahippocampal connectivity and neuroticism. Neuropsychopharmacology 2014;39:875-84

40. Samuels J, Nestadt G, Bienvenu OJ, et al. Personality disorders and normal personality dimensions in obsessive-compulsive disorder. Br J Psychiatry 2000;177:457-62.

41. Tang HS, Chen PF, Lung FW. Personality and alexithymic disparity in obsessive-compulsive disorder based on washing and checking. Psychiatr Q 2018;89:371-81.

42. Larsen RJ, Ketelaar T. Personality and susceptibility to positive and negative emotional states. J Pers Soc Psychol 1991;61:132-40.

43. Ng W, Diener E. Personality differences in emotions: does emotion regulation play a role? J Individ Differ 2009;30:100-6

44. Nicholson AA, Densmore M, Frewen PA, et al. The dissociative subtype of posttraumatic stress disorder: unique resting-state functional connectivity of basolateral and centromedial amygdala complexes. Neuropsychopharmacology 2015;40:2317.

45. Stenbacka L. Functional imaging of peripheral vision and dorsal stream function in the human cerebral cortex. Helsingin Yliopisto 2010;44:706-10.

46. Koelsch S, Skouras S, Fritz T, et al. The roles of superficial amygdala and auditory cortex in music-evoked fear and joy. Neuroimage 2013;81:49-60.

47. Fusar-Poli P, Placentino A, Carletti F, et al. Functional atlas of emotional faces processing: a voxel-based meta-analysis of 105 functional magnetic resonance imaging studies. J Psychiatry Neurosci 2009;34: 418-32.

48. Eckstein M, Markett S, Kendrick KM, et al. Oxytocin differentially alters resting state functional connectivity between amygdala subregions and emotional control networks: inverse correlation with depressive traits. Neuroimage 2017;149:458-67.

49. Phelps EA, LeDoux JE. Contributions of the amygdala to emotion processing: from animal models to human behavior. Neuron 2005;48:175-87.

50. Hrybouski S, Aghamohammadi-Sereshki A, Madan CR, et al. Amygdala subnuclei response and connectivity during emotional processing. Neuroimage 2016;133:98-110.

51. Pessoa L, Adolphs R. Emotion processing and the amygdala: from a "low road" to "many roads" of evaluating biological significance. Nat Rev Neurosci 2010;11:773-83.
52. Vuilleumier P. How brains beware: neural mechanisms of emotional attention. Trends Cogn Sci 2005;9:585-94.

53. Thorsen AL, Hagland P, Radua J, et al. Emotional processing in obsessive-compulsive disorder: a systematic review and metaanalysis of 25 functional neuroimaging studies. Biol Psychiatry Cogn Neurosci Neuroimaging 2018;3:563-71.

54. Pauls DL, Abramovitch A, Rauch SL, et al. Obsessive-compulsive disorder: an integrative genetic and neurobiological perspective. Nat Rev Neurosci 2014;15:410-24.

55. van den Heuvel OA, van Wingen G, Soriano-Mas C, et al. Brain circuitry of compulsivity. Eur Neuropsychopharmacol 2016;26:810-27.

56. De Gelder B, Terburg D, Morgan B, et al. The role of human basolateral amygdala in ambiguous social threat perception. Cortex 2014;52:28-34

57. Hortensius R, Terburg D, Morgan B, et al. The basolateral amygdalae and frontotemporal network functions for threat perception. eNeuro 2017;4:0314-6.

58. Fonzo GA, Ramsawh HJ, Flagan TM, et al. Cognitive-behavioral therapy for generalized anxiety disorder is associated with attenuation of limbic activation to threat-related facial emotions. J Affect Disord 2014;169:76-85.

59. Reinecke A, Thilo KV, Croft A, et al. Early effects of exposure-based cognitive behaviour therapy on the neural correlates of anxiety. Transl Psychiatry 2018;8:225.

60. Ochsner KN, Bunge SA, Gross JJ, et al. Rethinking feelings: an FMRI study of the cognitive regulation of emotion. J Cogn Neurosci 2002;14:1215-29.

61. Rohr CS, Villringer A, Solms-Baruth C, et al. The neural networks of subjectively evaluated emotional conflicts. Hum Brain Mapp 2016:37:2234-46

62. Yu X, Liu L, Chen $\mathrm{W}$, et al. Integrity of amygdala subregion-based functional networks and emotional lability in drug-nave boys with ADHD. J Atten Disord 2020;24:1661-73.

63. Shin DJ, Jung WH, He Y, et al. The effects of pharmacological treatment on functional brain connectome in obsessive-compulsive disorder. Biol Psychiatry 2014;75:606-14.

64. Klumpp H, Fitzgerald DA, Phan KL. Neural predictors and mechanisms of cognitive behavioral therapy on threat processing in social anxiety disorder. Prog Neuropsychopharmacol Biol Psychiatry 2013:45:83-91.

65. Klumpp H, Roberts J, Kennedy AE, et al. Emotion regulation related neural predictors of cognitive behavioral therapy response in social anxiety disorder. Prog Neuropsychopharmacol Biol Psychiatry 2017;75:106-12.

66. Whitfield-Gabrieli S, Ghosh SS, Nieto-Castanon A, et al. Brain connectomics predict response to treatment in social anxiety disorder. Mol Psychiatry 2016;21:680-5.

67. Reggente N, Moody TD, Morfini F, et al. Multivariate resting-state functional connectivity predicts response to cognitive behavioral therapy in obsessive-compulsive disorder. Proc Natl Acad Sci U S A 2018;115: 2222-7.

68. Mataix-Cols D, Rosario-Campos MC, Leckman JF. A multidimensional model of obsessive-compulsive disorder. Am J Psychiatry 2005;162:228-38 